\title{
Reduced protective effect of Plasmodium berghei immunization by concurrent Schistosoma mansoni infection
}

\author{
Ramon F Laranjeiras, Luísa CC Brant, Anna Carolina L Lima, \\ Paulo Marcos Zech Coelho, Erika M Braga/ ${ }^{+}$ \\ Departamento de Parasitologia, Instituto de Ciências Biológicas, Universidade Federal de Minas Gerais, Av. Antônio Carlos 6627, \\ 31270-901 Belo Horizonte, MG, Brasil
}

\begin{abstract}
Studies on concomitant schistosomiasis and human and experimental malaria have shown a variation in the immunospecific response, as well as an increase in the severity of both parasitoses. In the present study, a murine co-infection model was used to determine the effects of a co-infection with Schistosoma mansoni and Plasmodium berghei on the protective immunity acquired by repeated malarial infections and subsequent curative treatment with chloroquine. Our results have demonstrated that, compared to an infection with P. berghei only, the co-infection increases the malarial parasitaemia and decreases the survival rate. Indeed, mice that were immunized by infection and treatment with drug displayed no mortality whereas co-infected mice showed a reduced protective efficacy of immunization against $\mathrm{P}$. berghei (mortality $>60 \%$ ). Interestingly, this high mortality rate was not associated with high levels of parasitaemia. Our findings support the idea of a suppressive effect of a Schistosoma co-infection on the anti-malarial protection by immunization. This result reveals a possible drawback of the development of antimalarial vaccines, especially considering the wide endemic areas for both parasitoses.
\end{abstract}

Key words: Plasmodium berghei - Schistosoma mansoni - co-infection - immunity

Concomitant parasitic infections are common events in different regions worldwide. Malaria and schistosomiasis share common transmission areas in various tropical regions, especially on the African continent. The consequences of the association of malaria and schistosomiasis in humans have been extensively reported. Enhancement of splenomegaly in co-infections has been described since the 1960s (Whittle et al. 1969) and confirmed in recent studies. Thus, a recent crosssectional study has demonstrated that chronic exposure to malaria is associated with hepatosplenomegaly, even in the absence of Schistosoma mansoni infection (Wilson et al. 2007). However, most children infected with $S$. mansoni showed only light to moderate infection intensities, despite having a greater enlargement of the liver and spleen than children who did not have schistosomiasis. Furthermore, a recent epidemiological study conducted by the same research group has suggested a possible immunological mechanism for childhood hepatomegaly in areas where transmission of both S. mansoni and Plasmodium falciparum exists. It was proposed that specific Th2 cytokine responses against schistosome eggs were lower among children who were co-infected with $P$. falciparum than in children infected with schistosome only (Wilson et al. 2008). This would indicate poor regulation of the inflammatory response to schistosome eggs.

Financial support: FAPEMIG, CNPq

+ Corresponding author: embraga@icb.ufmg.br

Received 24 April 2008

Accepted 10 October 2008
Additionally, it has been shown that hepatosplenic children infected with $S$. mansoni have higher levels of anti- $P$. falciparum antibodies (IgG1 and IgG3) than nonhepatosplenic children (Mwatha et al. 2003). Moreover, a strong correlation between malaria and schistosomespecific $\operatorname{IgG} 3$ responses was observed in different populations living in areas where the two infections occur simultaneously (Naus et al. 2003). Furthermore, the crossreactivity between schistosome and malarial antigens has been proposed in human populations (Mutapi et al. 2007). Indeed, the authors have demonstrated that the levels of IgG1, IgG3 and IgG4 against malarial antigens are high at low schistosome infection intensities, but that they decrease with increasing schistosome infection intensity. Indeed, the authors have demonstrated that the levels of IgG1, IgG3 and IgG4 against malarial antigens are high at low schistosome infection intensities. Additionally, the levels of IgG2, IgG3 and IgG4 against malarial antigens are positively correlated with the levels of the same $\operatorname{IgG}$ subclasses against soluble schistosome egg antigens.

In contrast, previous study demonstrated an agespecific protective effect of Schistosoma hematobium infection following the acquisition of uncomplicated $P$. falciparum malaria (Lyke et al. 2005). Children with schistosomiasis have less severe malaria, their first clinical symptoms are delayed and parasitaemia is reduced.

It has also been shown in experimental models that co-infection with $S$. mansoni and murine Plasmodium spp. leads to a two-fold increase in parasitaemia, as compared to what is observed for a single malarial infection (Lwin et al. 1982). More recently, it has been reported that superinfection with $S$. mansoni enhances the development of Plasmodium berghei, increases both parasitaemia and mortality and delays the reduction/ 
clearance in parasitaemia (Legesse et al. 2004). Moreover, previous studies have revealed that mice co-infected with S. mansoni and Plasmodium chabaudi develop malarial parasitaemia in the blood more rapidly and at higher levels (Helmby et al. 1998). The authors have also demonstrated that two parasitic infections, namely the Th2-dominated S. mansoni infection and the acute Th1dependent blood-stage $P$. chabaudi infection, severely affect the immune response to each other when they are present simultaneously in experimental mice.

Considering that an $S$. mansoni infection can possibly render a malarial infection more potent, as previously described, we can hypothesize that the co-existence of the $S$. mansoni infection in endemic areas for malaria can impair the development of a protective immunity that is naturally acquired after exposure to the malarial parasite. This may be an important confounding factor that may impede the development of vaccine-induced protective immunity against malaria. In the present study, we have used a $P$. berghei-S. mansoni co-infection murine model to test the hypothesis that a concurrent $S$. mansoni infection impairs the immunization against lethal malaria by repeated blood-stage infections and chemotherapeutic drug treatments.

\section{MATERIAL AND METHODS}

Animals and experimental infections - Female Balb $\mathrm{C}$ mice, aged six weeks, and $S$. mansoni cercariae were obtained from the animal facility of the Schistosomiasis Research Unit, Institute of Biological Sciences, UFMG, Brazil. Rodent chow and water were given ad libitum. LE strain S. mansoni cercariae (Valadares et al. 1981) released from Biomphalaria glabrata snails were suspended in saline $0.9 \%$ and subcutaneously injected ( 20 cercariae per animal) by an experienced technician. $S$. mansoni infection started 24 weeks prior to $P$. berghei infection. Successful S. mansoni infection was confirmed by stool examination (sedimentation concentration method) for the detection of viable eggs.

The $P$. berghei NK 65 strain was obtained from the Laboratory of Malaria, UFMG. The parasite was maintained by intraperitoneal subinoculation of $10^{6}$ parasitized erythrocytes obtained from infected donor mice into clean mice at weekly intervals. Parasitaemia was evaluated daily by Giemsa staining of thin blood smears prepared with a drop of blood obtained from the tails of the mice. Parasitaemia was determined up to 30 days post-infection. The mice mortality was recorded daily.

Induction of malarial immunity by infection and drug treatment - P. berghei infection leads to fatal parasitaemia in mice. Resistance to the infection can be achieved when mice are allowed to recover from the blood-induced infection by administration of chloroquine. In Balb/c mice, at least two cycles of infection and subsequent drug treatment were necessary to establish immunity (no parasitaemia was detected after additional challenges after the third infection). Thus, mice were immunized against $P$. berghei by successive experimental infections $\left(10^{6}\right.$ parasitized erythrocytes), followed by radical cure after treatments with chloroquine $(100 \mathrm{mg} / \mathrm{kg}$, orally).
Infected mice received the anti-malarial treatment for four consecutive days, starting $24 \mathrm{~h}$ after the infectious inoculum. Ten days after the first inoculum, infection was repeated and followed by treatment. This procedure was successively repeated four times. The induction of immunity against malaria started 18 weeks after the $S$. mansoni infection.

Experimental groups - Mice were distributed into four groups: (1) 20 mice previously immunized against malaria with the drug treatment protocol and challenged with $10^{6}$ parasitized erythrocytes; (2) 16 mice chronically infected with $S$. mansoni and subsequently immunized with the drug treatment protocol (these animals were also further challenged with $10^{6}$ parasitized erythrocytes); (3) 15 mice infected with $P$. berghei (control group); and (4) 13 mice chronically infected with $S$. mansoni and subsequently infected with $P$. berghei.

Ethichal permission to conduct the study was obtained from the Committee of Animal Experimentation of the Universidade Federal de Minas Gerais (UFMG), Brazil.

Statistical analysis - Statistical analyses were carried out using the Program R version 2.2.1. for Windows. Survival data were estimated and according to the KaplanMeier Method. All statistical tests were two-sided; $p$ values less than 0.05 were considered statistically significant.

\section{RESULTS}

Parasitaemia in the different experimental groups - Balb C mice immunized against lethal malarial infection by repeated infections and drug treatments (group 1) showed the lowest levels of parasitaemia. Mice immunized with the drug treatment but co-infected with S. mansoni also displayed low levels of parasitaemia during the experimental period (group 2). Mice belonging to groups 3 and 4 died prematurely and high levels of parasitaemia were detected during the follow-up. For animals from group 4, only two co-infected mice had a survival time longer than 11 days. Animals from group 3 (control animals with $P$. berghei infection only) had longer survival times than those from group $4(\mathrm{p}<0.05)$. It was observed that the parasitaemia levels for immunized mice (groups 1 and 2) were lower than the levels obtained for non-immunized mice (groups 3 and 4) $(\mathrm{p}<0.05)$ (Fig. 1).

Reduced induction of immunization against malaria in S. mansoni infected mice - The effect of co-infection and immunization on the survival time of mice was evaluated. Figs $2 \mathrm{~A}$ and $2 \mathrm{~B}$ show the survival curves that were evaluated with the Kaplan-Meier Method for the co-infection and immunization, respectively. They illustrate the effect of these co-variables on the survival time of mice. The figures clearly demonstrate that the two analyzed co-variables (co-infection and immunization) are important for the survival of the mice. As depicted in Fig. 2A, the survival time after a P. berghei infection is significantly decreased in co-infected mice (group 4) when compared to those infected by P. berghei only (group 3). We have also analyzed the effect of coinfection on the acquisition of anti-malarial immunity. As shown in Fig. 2B, Schistosoma co-infection aggravates malaria in non-immunized mice. Indeed, mice 
from group 1 displayed no mortality whereas those from group 2 showed significant mortality (62\%). Interestingly, the high mortality occurs when the parasitaemia is very low (Fig. 1). This suggests that the co-infection does not impair or disrupt the anti-malarial immunity, but that it somehow triggers severe pathogenesis.

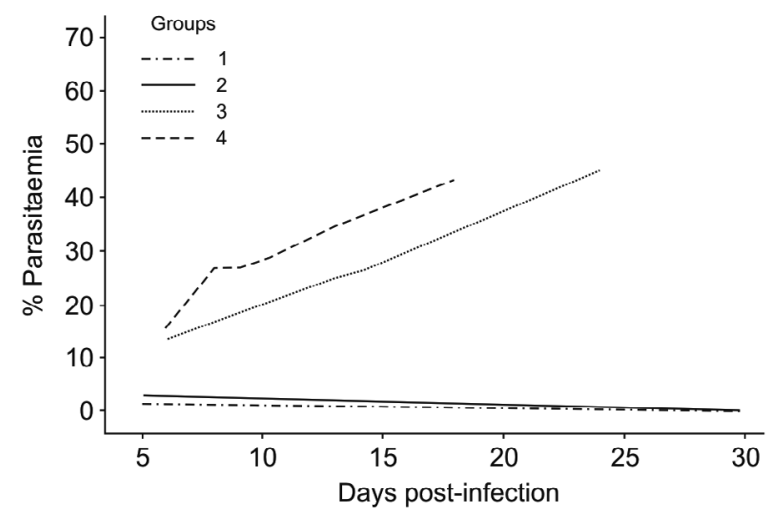

Fig. 1: graph of the smoothing curve (lowess) representing the mean parasitaemia values changing with time after Plasmodium berghei infection for the groups of mice experimentally infected groups: 1: mice infected with $P$. berghei and previously immunized against malaria by successive infections followed by treatment $(n=20) ; 2$ : mice chronically infected with Schistosoma mansoni, subsequently infected with $P$. berghei and previously immunized against malaria by successive infections followed by treatment $(\mathrm{n}=16) ; 3$ : mice infected with $P$. berghei $(\mathrm{n}=15) ; 4$ : mice chronically infected with $S$. mansoni and subsequently infected with $P$. berghei $(\mathrm{n}=13)$.

\section{DISCUSSION}

The association of $P$. falciparum with schistosomes and other helminthes in endemic areas constitutes a confounding factor in the assessment of the efficacy of malaria-control interventions, including vaccine prototypes in clinical trials (Druilhe et al. 2005). The influence of schistosomes in the acquisition of immunity against Plasmodium has been proposed in several field studies (Diallo et al. 2004, Sokhna et al. 2004, Mutapi et al. 2007). However, the actual findings are controversial as epidemiological observations suggest a range of scenarios in which helminth infections may not only increase the susceptibility to Plasmodium infections, but also protect against severe malaria under certain circumstances (reviewed in Nacher 2004, Brooker et al. 2007, Nacher 2008).

In this study, we have demonstrated that mice carrying a $S$. mansoni infection and infected with blood-stage $P$. berghei parasites manifest malaria more rapidly and severely. This indicates a failure in the initial immunological mechanism involved in parasite control. It could also be explained by the fact that both diseases cause pathologies with similar morbid impacts, such as anaemia. Indeed, similar to malaria, anaemia caused by schistosomes can arise from the destruction of red blood cells and/or dyserythropoiesis. Although in this study we did
A

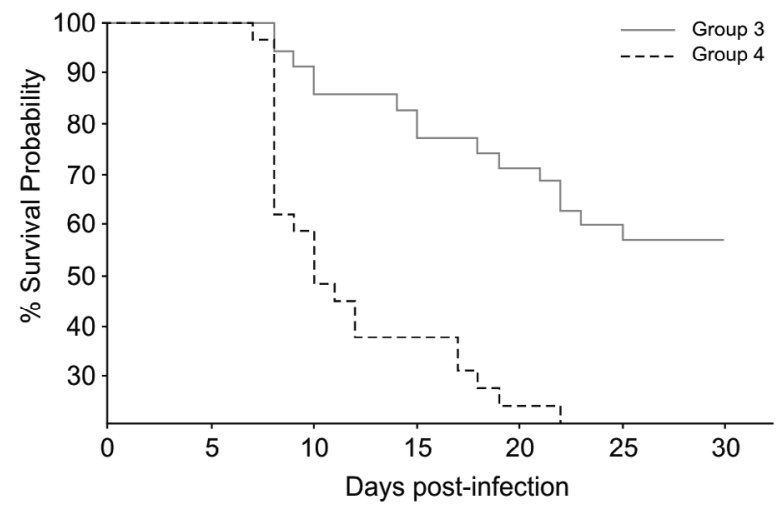

B

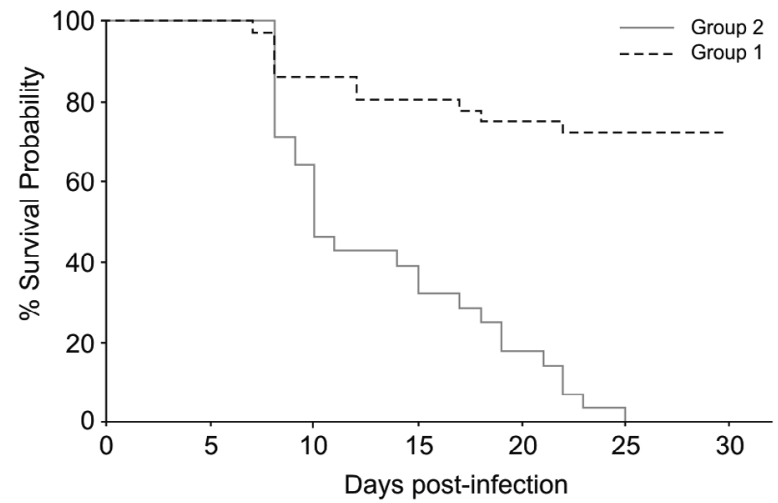

Fig. 2: survival curves assessed by means of the Kaplan-Meier Method for the malaria and schistosomiasis co-infection co-variable (A) and for the immunization co-variable (B).

not measure haemoglobin levels or other haematological parameters, it is possible to speculate that if these diseases were associated, the anaemia would be exacerbated because of the synergism of the effects of both parasites.

As previously mentioned, very little is known about the mechanisms by which the interactions between parasites occur and the experimental infection of mice with $P$. berghei and S. mansoni offers an appropriate model for the study of this association. In this study, we have established a murine model of the co-infection with both parasites in order to evaluate the effect of a concurrent helminth infection on the acquisition of the anti-malarial immunity. Thus, we have demonstrated that Schistosoma-free mice that are immunized via infection and drug treatment develop a strong protection against blood-stage malarial infection as indicated by the low level of parasitaemia and the high survival rate. Although immunized Schistosoma-infected mice also showed a low degree of parasitaemia, the survival rate was not improved. This lack of correlation suggests that co-infection does not impair or disrupt the anti-parasite immunity, but that it triggers severe pathogenesis. The effects of concomitant infections on the development, as well as the maintenance of an immune response remain unclear. However, it has been known that the successful 
resolution of a Plasmodium infection requires a coordinated succession from a Th1- to a Th2-type response, and that anything that upsets the timing or balance of this process can lead to a chronic or severe infection (Cox 2001, Brooker et al. 2007). Thus, we believe that a Th2-skewed immune profile induced by a chronic schistosomiasis might affect the course of the Plasmodium infection and the acquisition of the malarial immunity. Based on the $\mathrm{T}$ cell dichotomy, the concomitant infection could lead to synergistic or antagonistic $\mathrm{T}$ cell/cytokine responses that could either decrease or exacerbate the pathological effects. However, the potential immuno-regulatory mechanisms by which helminthes may alter their immune responses to malaria are still not understood.

Our findings illustrate the need for complementary studies in order to evaluate the mechanisms that could impair the acquisition of resistance against $P$. berghei in mice co-infected with $S$. mansoni. The present results allow us to infer that similar findings may be observed in human populations living in areas where the transmission of both of these two important parasitoses exists. Moreover, our results suggest that a schistosome treatment may be an effective strategy to improve the efficacy of malarial vaccines in humans living in areas where malaria and schistosomiasis coexist.

\section{REFERENCES}

Brooker S, Akhwale W, Pullan R, Estambale B, Clarke SE, Snow RW, Hotez PJ 2007. Epidemiology of Plasmodium-helminth co-infection in Africa: populations at risk, potential impact on anemia and prospects for combining control. Am J Trop Med Hyg 77: 88-98.

Cox FE2001. Concomitant infections, parasites and immune responses. Parasitology 122: 23-38.

Diallo TO, Remoue F, Schacht AM, Charrier N, Dompnier JP, Pillet S, Garraud O, N'Diaye AA, Capron A, Capron M, Riveau G 2004. Schistosomiasis co-infection in humans influences inflammatory markers in uncomplicated Plasmodium falciparum malaria. Parasite Immunol 26: 365-369.

Druilhe P, Tall A, Sokhna C 2005. Worms can worsen malaria: towards a new means to roll back malaria? Trends Parasitol 21: 359-362.

Helmby H, Kullberg M, Troye-Blomberg M 1998. Altered immune responses in mice with concomitant Schistosoma mansoni and Plasmodium chabaudi infections. Infect Immun 66: 5167-5174.

Legesse M, Erko B, Balcha F 2004. Increased parasitaemia and delayed parasite clearance in Schistosoma mansoni and Plasmodium berghei co-infection Acta Trop 91: 161-166.

Lwin M, Last C, Targett GA, Doenhoff MJ 1982. Infection of mice concurrently with Schistosoma mansoni and rodent malarias: con- trasting effects of patent $S$. mansoni infections on Plasmodium chabaudi, P. yoelii and P. berghei. Ann Trop Med Parasitol 76: 265-273.

Lyke KE, Dicko A, Dabo A, Sangare L, Kone A, Coulibaly D, Guindo A, Traore K, Daou M, Diarra I, Sztein MB, Plowe CV, Doumbo OK 2005. Association of Schistosoma haematobium infection with protection against acute Plasmodium falciparum malaria in Malian children. Am J Trop Med Hyg 73: 1124-1130.

Mutapi F, Roussilhon C, Mduluza T, Druilhe P 2007. Anti-malaria humoral responses in children exposed to Plasmodium falciparum and Schistosoma haematobium. Mem Inst Oswaldo Cruz 102: 405-409.

Mwatha JK, Jones FM, Mohamed G, Naus CW, Riley EM, Butterworth AE, Kimani G, Kariuki CH, Ouma JH, Koech D, Dunne DW 2003. Associations between anti-Schistosoma mansoni and anti-Plasmodium falciparum antibody responses and hepatosplenomegaly in Kenyan schoolchildren. J Inf Dis 187: 1337-1341.

Nacher M 2004. Interactions between worm infections and malaria. Clin Rev Allergy Immunol 26: 85-92.

Nacher M 2008. Worms and malaria: blind men feeling the elephant? Parasitology 135: 861-868.

Naus CW, Jones FM, Satti MZ, Joseph S, Riley EM, Kimani G, Mwatha JK, Kariuki CH, Ouma JH, Kabatereine NB, Vennervald BJ, Dunne DW 2003. Serological responses among individuals in areas where both schistosomiasis and malaria are endemic: cross-reactivity between Schistosoma mansoni and Plasmodium falciparum. J Inf Dis 187: 1272-1282.

Sokhna C, Le Hesran JY, Mbaye PA, Akiana J, Camara P, Diop M, Ly A, Druilhe P 2004. Increase of malaria attacks among children presenting concomitant infection by Schistosoma mansoni in Senegal. Malar J 3: 43.

Valadares TE, Coelho PM, Pellegrino J, Sampaio IB 1981. Schistosoma mansoni: comparison of oviposition of the LE' (Belo Horizonte), SP (Sao Paulo) and ST (Liberia) strains in mice. Rev Inst Med Trop Sao Paulo 23: 1-5.

Whittle H, Gelfand M, Sampson E, Purvis A, Weber M 1969. Enlarged livers and spleens in an area endemic for malaria and schistosomiasis. Trans R Soc Trop Med Hyg 63: 353-361.

Wilson S, Jones FM, Mwatha JK, Kimani G, Booth M, Kariuki HC, Vennervald BJ, Ouma JH, Muchiri E, Dunne DW 2008. Childhood schistosomiasis and malaria co-infection: hepatosplenomegaly is associated with low regulatory and $\mathrm{Th} 2$ responses to schistosome antigens. Infect Immun 76: 2212-2218.

Wilson S, Vennervald BJ, Kadzo H, Ireri E, Amaganga C, Booth M, Kariuki HC, Mwatha JK, Kimani G, Ouma JH, Muchiri E, Dunne DW 2007. Hepatosplenomegaly in Kenyan schoolchildren: exacerbation by concurrent chronic exposure to malaria and Schistosoma mansoni infection. Trop Med Int Health 12: 1442-1449. 